viduelle Stimmverhalten gesunken ist. Im Gegenzug sollten kurzfristige politische Orientierungen - unter anderem auch der Einfluss bundespolitischer Konstellationen - an Einfluss gewonnen haben.

(4) Auf der Aggregatebene lassen sich deutliche Einflüsse der Bundes- und der Landespolitik auf das Abschneiden von CDU, SPD, FDP, Bündnis 90/Die Grünen sowie der sonstigen Parteien bei rheinland-pfälzischen Landtagswahlen erkennen. Parteien, die im Bund regieren, schneiden bei Landtagswahlen systematisch schlechter ab als Parteien, die in Bonn beziehungsweise Berlin die Opposition stellen. Mit Ausnahme der FDP führt eine Regierungsbeteiligung im Land hingegen zu überdurchschnittlich guten Wahlergebnissen. Im Allgemeinen fällt jedoch das Gewicht bundespolitischer Faktoren größer aus als der Einfluss landespolitischer Konstellationen.

(5) Auf der Individualebene ist dieses Muster nicht zu erkennen. Zum einen ist die Bedeutung von Bundes- und Landespolitik für das Landtagswahlverhalten der RheinlandPfälzer gering; die Stimmenabgabe wird nach wie vor vor allem von der Parteibindung beeinflusst. Zum anderen ist weder ein generelles Übergewicht bundespolitischer noch eine systematisch größere Bedeutung landespolitischer Faktoren zu erkennen. Dies gilt über alle untersuchten rheinland-pfälzischen Landtagswahlen und Parteien hinweg.

\title{
Landtagswahlen in Bayern 1966 bis 2003: verstärkte bundespolitische Durchdringung aufgrund der Doppelrolle der CSU
}

\section{Harald Schoen}

Den Bayern ist es besser als den Bürgern anderer Bundesländer gelungen, die Aufmerksamkeit der häufig am Verhalten des deutschen Durchschnittswählers interessierten Wahlforschung auf sich zu ziehen. Eindrücklichstes Beispiel dafür ist die Kontroverse zwischen Jürgen W. Falter und Alf Mintzel in dieser Zeitschrift über die Besonderheiten der bayerischen Wähler. ${ }^{1}$ Obgleich darin spezifisch bayerisches Wahlverhalten vermutet wurde, ging die Forschung nicht so weit anzunehmen, Bayerns Bürger würden die Bundespolitik vollkommen außer Acht lassen, wenn sie über die Zusammensetzung des Landtages entscheiden, würden also in diesem Sinn unverwechselbar bayerisch wählen. Im Gegenteil, zahlreiche Analysen gehen ausdrücklich von bundespolitischen Einflüssen auf bayerisches Landtagswahlverhalten aus. So habe die CSU in den siebziger Jahren „uneingeschränkt von einem aus der Dialektik zwischen Bundes- und Landtagswahlen folgenden, Oppositionsef-

1 Siehe Jürgen W. Falter, Bayerns Uhren gehen wirklich anders. Politische Verhaltens- und Einstellungsunterschiede zwischen Bayern und dem Rest der Republik, in: ZParl, 13. Jg. (1982), H. 4, S. 504 - 521; Alf Mintzel, Gehen Bayerns Uhren wirklich anders?, in: ZParl, 18. Jg. (1987), H. 1, S. 77 - 93; Jürgen W. Falter, Wie gehen sie denn nun wirklich, die bayerischen Uhren?, in: ZParl, 19. Jg. (1988), H. 1, S. 113 f. 
fekt'“2 profitiert. In dieser Zeit hätten gerade bundespolitische Einflüsse wie die Deutschland- und Ostpolitik der sozial-liberalen Koalition entscheidend zum Vordringen der CSU in die protestantischen Teile Frankens beigetragen und damit zur elektoralen Homogenisierung Bayerns zu einer „geschlossenen Gesamt-Hochburg der CSU“3. Auch bei der Landtagswahl 1994 sei die CSU vom Bundestrend getragen worden ${ }^{4}$, wie sie 2003 von der verbreiteten Unzufriedenheit mit der rot-grünen Bundesregierung profitiert habe. ${ }^{5}$ Folgt man diesen Einschätzungen, scheinen selbst die weithin als bayerische Besonderheit anerkannten regelmäßigen absoluten CSU-Mehrheiten bei Landtagswahlen kein allein mit landespolitischen Faktoren erklärbares Phänomen.

Mögen diese Aussagen plausibel erscheinen, so handelt es sich doch eher um Vermutungen als um gesicherte Erkenntnisse, da sie nicht auf empirischen Analysen individuellen Landtagswahlverhaltens beruhen. Der vorliegende Beitrag soll helfen, diese Lücke in der Forschung zu schließen.

\section{Landes- und bundespolitische Faktoren bei bayerischen Landtagswahlen}

Landtagswahlverhalten lässt sich mit dem bei der Analyse von nationalen Wahlen sehr gut bewährten sozialpsychologischen Modell erklären. Demnach kann man die Wahlentscheidung vereinfacht auffassen als eine Resultante aus langfristiger Parteiidentifikation sowie kurzfristig variablen Orientierungen gegenüber politischen Akteuren und Streitfragen. ${ }^{6}$ Angewandt auf Landtagswahlen sind neben ebenenunspezifischen Parteibindungen Einstellungen zu Landespolitikern, etwa Spitzenkandidaten, sowie zu Sachfragen, seien es Kompetenzzuweisungen an Parteien zu spezifischen Themen, seien es generalisierte Urteile über die Leistung von Regierung und Opposition, zu berücksichtigen. Zudem können bundespolitische Orientierungen eine Rolle spielen. Neben der Möglichkeit, Wirkungen auf die parteipolitische Konsonanz von Bundesrat und Bundestag als Kriterium beim Landtagsvotum zu verwenden, sind vor allem Einstellungen zu Sachfragen und Politikern der Bundesebene zu beachten. ${ }^{7}$

2 Gabriele Jung / Günter Rieger, Die bayerische Landtagswahl vom 25. September 1994: Noch einmal gelang der CSU ein machiavellisches Lehrstück, in: ZParl, 26. Jg. (1995), H. 2, S. 232 -249 , S. 241.

3 Alf Mintzel, Gehen Bayerns Uhren wirklich anders?, a.a.O., S. 89 (Hervorhebung im Original); vgl. auch ders., Die bayerische Landtagswahl vom 27. Oktober 1974. Triumph einer konservativen Partei: ein wahlsoziologischer Sonderfall?, in: ZParl, 6. Jg. (1975), H. 4, S. $429-446$, S. 434.

4 Vgl. Gabriele Jung / Günter Rieger, a.a.O., S. 241.

5 Vgl. Günter Rieger, Die bayerische Landtagswahl vom 21. September 2003: Banale Sensationen, in: ZParl, 34. Jg. (2003), H. 4, S. $702-720$, S. 719.

6 Vgl. die Ausführungen von Oscar W. Gabriel und Everhard Holtmann zu dem Modell in diesem Heft der ZParl; vgl. auch Angus Campbell / Philip E. Converse / Warren E. Miller / Donald E. Stokes, The American Voter, New York 1960.

7 Vgl. Harald Schoen, Mir san mir an der weiß-blauen Wahlurne? Eine Analyse des Einflusses der Bundespolitik auf Wahlverhalten bei bayerischen Landtagswahlen 1966 bis 2003, in: Kerstin Völkl / Kai-Uwe Schnapp / Oscar W. Gabriel / Everhard Holtmann (Hrsg.), Wähler und Landtagswahlen in der Bundesrepublik Deutschland, Baden-Baden 2007 (i.E.). 
Mit Blick auf den Wahlausgang sind hinsichtlich dieser potentiellen Einflussfaktoren zwei Fragen von Bedeutung: Zum einen ist zu klären, wie sie verteilt sind, zum anderen, welche Merkmale wie stark auf individuelles Wahlverhalten wirken. Wirkungslose Größen sind naturgemäß uninteressant für den Wahlausgang. Aber selbst auf der Individualebene einflussreiche Faktoren können den Wahlausgang unberührt lassen, wenn ihre Verteilung in der Bevölkerung nicht von dem Wahlergebnis abweicht, das ohne ihren Einfluss zustande käme. Im Folgenden wird zunächst die Verteilung von Parteibindungen, landespolitischen Einstellungen sowie bundespolitischen Orientierungen vor bayerischen Landtagswahlen 1966 bis 2003 dokumentiert, ehe die Wirkung bundespolitischer Orientierungen auf das Landtagswahlverhalten untersucht wird. ${ }^{8}$

Parteibindungen sind in Bayern spätestens seit Beginn der 1980er Jahre asymmetrisch zugunsten der CSU verteilt (vgl. Tabelle 1). Vor der Wahl 1974 hielten sich SPD- und CSU-Anhänger mit je einem Drittel die Waage. Während die Zahl der CSU-Identifizierer seitdem leicht anstieg, ist sie bei der SPD deutlich gesunken. Im Ergebnis sind seit 1982 vor Landtagswahlen rund doppelt so viele CSU- wie SPD-Anhänger zu finden. Der Anteil der Sympathisanten anderer Parteien blieb seit 30 Jahren praktisch stabil. Im Einklang mit allgemeinen Tendenzen des Dealignment wuchs dagegen die Zahl der Parteilosen merklich an. ${ }^{9}$ Insgesamt verschafft die Verteilung langfristiger Parteiloyalitäten der CSU einen deutlichen Wettbewerbsvorteil bei Landtagswahlen.

\begin{tabular}{|c|c|c|c|c|}
\hline Tabelle 1: Parteibindungen in Bayern, 1974 bis 2003 (Angaben in Prozent) \\
\hline & CSU & SPD & andere Partei & $\begin{array}{c}\text { keine Partei- } \\
\text { identifikation }\end{array}$ \\
\hline 1974 & 31 & 32 & 16 & 21 \\
\hline 1982 & 40 & 23 & 14 & 23 \\
\hline 1986 & 37 & 16 & 15 & 32 \\
\hline 1990 & 38 & 19 & 11 & 32 \\
\hline 1994 & 34 & 19 & 14 & 33 \\
\hline 1998 & 34 & 19 & 10 & 37 \\
\hline 2003 & 41 & 16 & 13 & 30 \\
\hline $\begin{array}{l}\text { Für 1966, 1970 und 1978 liegen keine entsprechenden Daten vor. } \\
\text { Quelle: Zentralarchiv Köln, ZA-Nr. 871, 1247, 1551, 1963, 2506, 3167, 3955. }\end{array}$ \\
\hline
\end{tabular}

Vor dem Hintergrund der asymmetrischen Verteilung der Parteibindungen erscheint es folgerichtig, dass Bayerns Bürger den Spitzenkandidaten der CSU und der Leistung der Staatsregierung in der Regel ein besseres Zeugnis ausstellen als SPD-Bewerbern und der Arbeit der sozialdemokratischen Landtagsopposition (vgl. Tabelle 2). Während die bayerische Staatsregierung seit nunmehr vierzig Jahren konstant gute Noten erhält, wird die SPD-Op-

8 Als Datengrundlage dienen Umfragen zu den Landtagswahlen 1966, 1974, 1982 bis 2003, die kurz vor dem jeweiligen Urnengang durchgeführt wurden (ZA-Nr. 472, 871, 1247, 1551, 1963 , 2506, 3167, 3955). Die Daten zur Wahl 1974 wurden im Frühsommer 1973 erhoben, weshalb bei ihrer Interpretation besondere Vorsicht geboten ist.

9 Siehe hierzu etwa Harald Schoen / Cornelia Weins, Der sozialpsychologische Ansatz in der empirischen Wahlforschung, in: Jürgen W. Falter / Harald Schoen (Hrsg.), Handbuch Wahlforschung, Wiesbaden 2005, S. 185 - 241, S. 222 ff. Der hohe CSU-Anteil 2003 läuft dem Trend zum Dealignment zuwider und könnte auf Mess- oder Stichprobenprobleme hindeuten. 
position im Maximilianeum dagegen eher mittelmäßig bewertet und fällt damit zeitweise dramatisch hinter die Regierung zurück. Größeren Schwankungen unterliegt das Urteil der Bayern über die Spitzenkandidaten der beiden großen Parteien. 1966 ließ Alfons Goppel mit dem höchsten Popularitätswert Volkmar Gabert von der SPD klar hinter sich. Vor der Wahl 1974 hatte sich Goppels Vorsprung auf Hans-Jochen Vogel deutlich verringert. Franz Josef Strauß konnte 1982 zwar Helmut Rothemund distanzieren, aber seinem Vorgänger nicht das Wasser reichen. Bis 1986 stieg seine Beliebtheit deutlich an, während Karl-Heinz Hiersemann ebenso mäßig beurteilt wurde wie sein Vorgänger. 1990 zog Max Streibl für die CSU in die Landtagswahl und konnte den Popularitätsvorsprung noch leicht ausbauen. Was Hans-Jochen Vogel nicht geglückt war, gelang 1994 Renate Schmidt: Als SPD-Herausforderin zog sie in der Beliebtheit mit dem frisch gewählten Ministerpräsidenten Edmund Stoiber praktisch gleich. Obgleich Stoiber danach leicht zulegte, konnte er auch bei der Wahl 1998 seine Kontrahentin in der Wählergunst nicht deutlich hinter sich lassen. Gerade bei diesen beiden Wahlen durfte sich daher die SPD von starken Kandidateneffekten einen deutlichen Stimmenbonus im Vergleich zu der sehr ungleichen Verteilung der Parteiloyalitäten versprechen. Anders 2003, als Franz Maget kaum beliebter war als seine Vorgänger Rothemund und Hiersemann und von Stoiber in punkto Popularität um Längen geschlagen wurde. Abgesehen von wenigen Ausnahmen genoss die CSU also auch bei landespolitischen Orientierungen einen deutlichen Bonus. ${ }^{10}$

\begin{tabular}{|c|c|c|c|c|}
\hline & $\begin{array}{c}\text { CSU- } \\
\text { Kandidat }\end{array}$ & $\begin{array}{c}\text { SPD- } \\
\text { Kandidat }\end{array}$ & $\begin{array}{c}\text { CSU- } \\
\text { Regierung }\end{array}$ & $\begin{array}{c}\text { SPD- } \\
\text { Opposition }\end{array}$ \\
\hline 1966 & 0,76 & 0,63 & 0,68 & - \\
\hline 1974 & 0,73 & 0,67 & 0,70 & - \\
\hline 1982 & 0,60 & 0,51 & 0,63 & 0,50 \\
\hline 1986 & 0,70 & 0,52 & 0,65 & 0,50 \\
\hline 1990 & 0,72 & 0,52 & 0,68 & 0,57 \\
\hline 1994 & 0,68 & 0,67 & 0,63 & 0,55 \\
\hline 1998 & 0,73 & 0,66 & 0,66 & 0,58 \\
\hline 2003 & 0,70 & 0,55 & 0,68 & 0,48 \\
\hline \multicolumn{5}{|c|}{$\begin{array}{l}\text { Der Wertebereich der Variablen erstreckt sich von } 0 \text { bis 1, wobei höhere Werte positivere Urteile indi- } \\
\text { zieren. } \\
\text { Für } 1970 \text { und } 1978 \text { liegen keine entsprechenden Daten vor. } \\
\text { Quelle: Zentralarchiv Köln, ZA-Nr. 472, 871, 1247, 1551, 1963, 2506, 3167, } 3955 .\end{array}$} \\
\hline
\end{tabular}

Die bundespolitischen Einstellungen sind im Vergleich dazu weniger eindeutig und konsistent zugunsten der CSU verteilt (vgl. Tabelle 3). Zweifelsohne muten 1966 die bundespolitischen Orientierungen beinahe wie ein Spiegelbild der landespolitischen Einstellungen an. Doch schon die Daten zur Wahl 1974, die allerdings im Frühsommer 1973 erhoben wurden und daher mit Vorsicht zu interpretieren sind, sahen ganz anders aus: Der noch bis Mai 1974 amtierende Bundeskanzler Willy Brandt war ähnlich populär wie die beiden Landeskandidaten, und der Bonner Oppositionsführer und Bundesvorsitzende der CDU Rainer Barzel sowie Franz Josef Strauß wurden von den Bayern mit so großer Reserve wie sonst al-

10 Dies gilt 1986 bis 2003 auch für landespolitische Kompetenzzuschreibungen. 
lenfalls Landespolitiker der SPD betrachtet. Folglich musste die CSU von bundespolitischen Effekten auf das Landtagswahlverhalten Stimmeneinbußen befürchten. Auch 1986 konnte sich die CSU von bundespolitischen Einflüssen keine großen Stimmenzuwächse erhoffen. Zwar war Johannes Rau lediglich so beliebt wie Hiersemann, doch stießen Helmut Kohl und seine Bundesregierung auf weniger Sympathie als Strauß und die bayerische Staatsregierung. Bis zur Wahl 1990 konnte Kohl seine Popularität und die seiner Regierung in Bayern deutlich steigern und seinen Herausforderer Oskar Lafontaine deutlich distanzieren. Noch etwas beliebter war Theo Waigel, Strauß' Nachfolger als CSU-Vorsitzender. Die Werte vor der Wahl 1994 entsprechen recht genau dem Muster des Jahres 1986. Dagegen ähnelt die Konstellation 1998 derjenigen vor der Wahl 1974. Helmut Kohl, Theo Waigel und die Bundesregierung wurden von den Bayern schlechter bewertet als Gerhard Schröder, dessen Ansehen allerdings weder an jenes von Willy Brandt 1973 noch an das von Renate Schmidt heranreichte. Daher musste die CSU befürchten, durch Bundeseinflüsse Stimmen zu verlieren. 2003 schließlich schnitt die rot-grüne Bundesregierung im Urteil der Bayern noch schlechter ab als ihre Vorgängerin und als sozialdemokratische Akteure in Bayern. Folglich konnten sich Bundeseinflüsse in diesem Fall als Belastung für die bayerische SPD erweisen.

\begin{tabular}{|c|c|c|c|c|}
\hline \multicolumn{5}{|c|}{$\begin{array}{l}\text { Tabelle 3: Einstellungen der bayerischen Bürger zu Bundespolitikern und zur Bundesregierung, } \\
1966 \text { bis } 2003\end{array}$} \\
\hline & Bundesregierung & CDU-Politiker & SPD-Politiker & CSU-Politiker \\
\hline 1966 & - & 0,71 & 0,56 & 0,63 \\
\hline 1974 & - & 0,51 & 0,67 & 0,52 \\
\hline 1986 & 0,61 & 0,59 & 0,52 & - \\
\hline 1990 & 0,67 & 0,69 & 0,56 & 0,71 \\
\hline 1994 & 0,57 & 0,60 & 0,51 & - \\
\hline 1998 & 0,52 & 0,51 & 0,59 & 0,52 \\
\hline 2003 & 0,41 & - & - & - \\
\hline \multicolumn{5}{|c|}{$\begin{array}{l}\text { Der Wertebereich der Variablen erstreckt sich von } 0 \text { bis 1, wobei höhere Werte positivere Urteile indi- } \\
\text { zieren. } \\
\text { Für 1970, } 1978 \text { und } 1982 \text { liegen keine entsprechenden Daten vor. } \\
\text { Quelle: Zentralarchiv Köln, ZA-Nr. 472, 871, 1551, 1963, 2506, 3167, } 3955 .\end{array}$} \\
\hline
\end{tabular}

Alles in allem genoss die CSU bei vielen der betrachteten Landtagswahlen im Hinblick auf Parteibindungen sowie landespolitische und bundespolitische Einstellungen gleichermaßen einen deutlichen Platzvorteil. In zumindest zwei Fällen (1974 und 1998) weicht das bundespolitische Bild jedoch deutlich vom landespolitischen ab. Gerade, aber nicht nur in diesen beiden Fällen ist es daher interessant zu wissen, ob bundespolitische Orientierungen bayerisches Landtagswahlverhalten beeinflussten.

\section{Der Einfluss bundespolitischer Orientierungen auf das bayerische Landtagswahlverhalten}

Die Frage, inwieweit bundespolitische Orientierungen unter statistischer Kontrolle anderer relevanter Faktoren die Wahlentscheidung beeinflussen, kann man mit Hilfe der multinominalen logistischen Regression beantworten. Sie gestattet es, die Wahl auf eine Reihe von Einflussgrößen zurückzuführen und deren Wirkung zu bestimmen. Im vorliegenden Fall wurde die Wahlentscheidung zwischen CSU, SPD, anderen Parteien und der Nichtwahl 
mit ebenenunspezifischen Parteibindungen und landespolitischen Faktoren erklärt. ${ }^{11}$ Hinzu kommen bundespolitische Orientierungen, und zwar Einstellungen zur Bundesregierung, zu Bundespolitikern sowie zu bundespolitischen Streitfragen. An dieser Stelle werden ausschließlich die zentralen Befunde zur Rolle der Bundespolitik dokumentiert.

Einstellungen zur Arbeit der Bundesregierung beeinflussten das Verhalten bei bayerischen Landtagswahlen nur tendenziell und zudem nicht immer nach dem prima facie erwarteten Muster, dass eine Landespartei aus positiven Bewertungen einer von ihr mitgetragenen Bundesregierung und aus negativen Urteilen über eine politisch konträre Bundesregierung Nutzen zieht. Bei den Landtagswahlen 1994 und 2003 ist diese Tendenz zu erkennen (vgl. Tabelle 4). 1986, 1990 und 1998 hingegen profitierten SPD und die kleinen Parteien der Tendenz nach auf Kosten der CSU von positiven Urteilen über die Arbeit der schwarz-gelben Bundesregierung. Diese Befunde deuten darauf hin, dass es der CSU mit ihrer Strategie, auf Bundesebene als innerkoalitionäre Opposition zu agieren, gelungen ist, Kritik an der von ihr gestützten Bundesregierung auf ihre Mühlen zu lenken. Angesichts der insgesamt schlechten Noten der schwarz-gelben Bundesregierung (vgl. Tabelle 3) scheint diese Strategie die bayerische Regierungspartei gerade 1998 vor Stimmenverlusten bewahrt zu haben.

\begin{tabular}{|c|c|c|c|c|c|}
\hline \multicolumn{6}{|c|}{$\begin{array}{l}\text { Tabelle 4: Wahrscheinlichkeit der Entscheidung für CSU, SPD, andere Parteien oder Nichtwahl } \\
\text { bei bayerischen Landtagswablen in Abhängigkeit von der Zufriedenheit mit der } \\
\text { Bundesregierung, } 1986 \text { bis } 2003\end{array}$} \\
\hline Wahl & $\begin{array}{c}\text { Bewertung der } \\
\text { Bundesregierung }\end{array}$ & CSU & SPD & Andere & Nichtwahl \\
\hline 1986 & $\begin{array}{l}\text { negativ } \\
\text { positiv }\end{array}$ & $\begin{array}{l}42 \\
34\end{array}$ & $\begin{array}{r}4 \\
21\end{array}$ & $\begin{array}{r}1 \\
15\end{array}$ & $\begin{array}{l}53 \\
30\end{array}$ \\
\hline 1990 & $\begin{array}{l}\text { negativ } \\
\text { positiv }\end{array}$ & $\begin{array}{l}33 \\
22 \\
\end{array}$ & $\begin{array}{r}6 \\
25 \\
\end{array}$ & $\begin{array}{l}25 \\
43^{*}\end{array}$ & $\begin{array}{l}35 \\
10 \\
\end{array}$ \\
\hline 1994 & $\begin{array}{l}\text { negativ } \\
\text { positiv }\end{array}$ & $\begin{array}{l}18 \\
24\end{array}$ & $\begin{array}{l}23 \\
22\end{array}$ & $\begin{array}{l}50 \\
37\end{array}$ & $\begin{array}{r}9 \\
17 \\
\end{array}$ \\
\hline 1998 & $\begin{array}{l}\text { negativ } \\
\text { positiv }\end{array}$ & $\begin{array}{l}46 \\
21\end{array}$ & $\begin{array}{l}20 \\
36\end{array}$ & $\begin{array}{l}30 \\
36\end{array}$ & $\begin{array}{l}4 \\
7\end{array}$ \\
\hline 2003 & $\begin{array}{l}\text { negativ } \\
\text { positiv }\end{array}$ & $\begin{array}{l}40 \\
25 \\
\end{array}$ & $\begin{array}{l}12 \\
22 \\
\end{array}$ & $\begin{array}{l}18 \\
33 \\
\end{array}$ & $\begin{array}{l}31 \\
21 \\
\end{array}$ \\
\hline \multicolumn{6}{|c|}{$\begin{array}{l}\text { * Die Differenz zwischen den Wahlwahrscheinlichkeiten für die Extremwerte der unabhängigen Variab- } \\
\text { len ist auf dem 95-Prozent-Niveau statistisch signifikant. } \\
1986 \text { entschieden sich } 42 \text { Prozent der Befragten ohne Parteibindung, die die Arbeit der Bundesregie- } \\
\text { rung sehr negativ bewerteten, für die CSU. Für Personen ohne Parteibindung, die die Arbeit der Bun- } \\
\text { desregierung sehr positiv beurteilten, beträgt der entsprechende Anteil 34 Prozent (siehe Fußnote 11). } \\
\text { Für 1966, 1970, 1974, } 1978 \text { und } 1982 \text { liegen keine für die Fragestellung nutzbaren Daten vor. } \\
\text { Quelle: Zentralarchiv Köln, ZA-Nr. 1551, 1963, 2506, 3167, 3955. }\end{array}$} \\
\hline
\end{tabular}

11 Im Einzelnen wurden folgende Variablen verwendet: Dummyvariablen für Anhänger von CSU, SPD und anderen Parteien, Einstellung zu den Landes-Spitzenkandidaten von CSU und SPD, Einstellungen zur Arbeit der Staatsregierung und der SPD-Opposition im Bayerischen Landtag, Kompetenzzuweisung an CSU und SPD, Einstellungen zu spezifischen Landesissues (1982), die wahrgenommene Wahrscheinlichkeit eines CSU-Wahlsieges und die Einstellung zu einer CSUAlleinregierung. Die berichteten Wahlwahrscheinlichkeiten wurden aus den multinomialen Logitmodellen für nicht parteigebundene Personen ermittelt, wobei alle außer der jeweils betrachteten Variable auf ihren Modus, Median oder ihr arithmetisches Mittel gesetzt wurden. 
Bei der Wahl 1982 kann die Wirkung konkreter bundespolitischer Sachfragen auf die Landtagswahlentscheidung untersucht werden. Dabei gingen von Einstellungen zur Einführung einer Ergänzungsabgabe sowie zur atomaren Nachrüstung kaum Effekte aus. Etwas stärker wirkten Urteile über das Scheitern der sozial-liberalen Koalition. Sah eine Person die Schuld bei der FDP, entschied sie sich in Bayern deutlich seltener für eine der unter der Rubrik „Andere“ zusammengefassten Parteien und stimmte stattdessen für die SPD oder blieb der Wahlurne fern. Wie ergänzende Analysen zeigen, scheinen diese bundespolitischen Einflüsse die FDP und die Grünen unter der Fünfprozentmarke gehalten und die Wahlchancen der SPD gesteigert zu haben, was frühere Vermutungen bestätigt. ${ }^{12}$ Die Machtfrage in Bayern, also die Mehrheit für die CSU, ließ jedoch auch dieses Issue unberührt.

Urteile über führende Bundespolitiker von CDU und SPD beeinflussten das Verhalten bei bayerischen Landtagswahlen generell nach einem plausiblen Muster: Eine günstige Meinung über einen CDU-Bundespolitiker machte die CSU-Wahl im Land wahrscheinlicher, während positive Einstellungen zu SPD-Bundesakteuren bayerische Wähler stärker dazu neigen ließ, für die SPD im Land zu stimmen (vgl. Tabelle 5). Allerdings gilt dies nicht bei allen betrachteten Wahlen in gleichem Maße. 1966 steigerte Wertschätzung für den Franken Ludwig Erhard die Wahrscheinlichkeit einer Stimmenabgabe für die CSU beträchtlich. Hatten 1966 Urteile über Willy Brandt das bayerische Wahlverhalten noch weniger beeinflusst als Bewertungen seines CDU-Pendants, wirkten sie 1974 - hier ist wieder besondere Vorsicht bei der Interpretation geboten - wesentlich stärker auf das Wahlverhalten als Meinungen über Rainer Barzel. Von Brandts Ansehen konnte sich die Landes-SPD Stimmengewinne versprechen. 1986 und 1990 begünstigten positive Einstellungen zu Helmut Kohl besonders die Entscheidung zugunsten der CSU, und zwar vor allem zu Lasten der FDP und anderer kleiner Parteien. ${ }^{13}$ Zustimmung zu Johannes Rau steigerte 1986 etwas die Wahrscheinlichkeit, für die SPD zu stimmen, vor allem begünstigte sie aber die Wahlenthaltung. 1990 hingegen schmälerten positive Urteile über Oskar Lafontaine die Chancen der CSU - und zwar zugunsten der SPD, der anderen Parteien, hier vor allem der Grünen, und der Nichtwahl in etwa gleichem Maße. Während Urteile über Kohl bei der Landtagswahl 1994 nur schwach wirkten, steigerten vorteilhafte Meinungen über Rudolf Scharping die SPD-Chancen zu Lasten der anderen Parteien und der Wahlenthaltung merklich. Ganz anders stellte sich die Situation 1998 dar: Nun beeinflussten Einstellungen zu den beiden Spitzenkandidaten für die zwei Wochen später stattfindende Bundestagswahl die Entscheidung zwischen CSU und SPD, was für Helmut Kohl noch stärker gilt als für Gerhard Schröder. Angesichts von Schröders Popularitätsvorsprung war dieser Kohl-Effekt jedoch für die CSU nicht unbedingt von Vorteil.

Einstellungen zu CSU-Vorsitzenden ohne Regierungsposition in Bayern spielten bei den betrachteten Wahlen eine nicht zu vernachlässigende Rolle. Wie den Ergebnissen in Tabelle 5 zu entnehmen ist, gewannen Urteile über Straußvon 1966, kurz vor seiner Amtszeit als Bundesfinanzminister, bis 1973, als er im Bundestag an vorderster Front die sozial-liberale

12 Siehe Jürgen W. Falter, Die bayerische Landtagswahl vom 10. Oktober 1982: Im Süden - beinahe - alles beim Alten, in: ZParl, 14. Jg. (1983), H. 1, S. 82 - 95, S. 94.

13 Eine ergänzende Analyse zeigt, dass 1990 positive Einstellungen zu Hans-Dietrich Genscher die Wahlentscheidung zugunsten der FDP begünstigte und Genschers Ansehen wesentlich zur einmaligen Rückkehr der Liberalen in das Maximilianeum beigetragen haben dürfte. 


\begin{tabular}{|c|c|c|c|c|c|c|c|c|c|c|c|c|c|c|}
\hline \multirow{2}{*}{\multicolumn{2}{|c|}{ 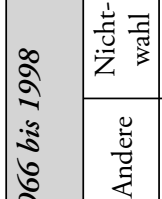 }} & $\tilde{m}$ & $\stackrel{\sim}{2}$ & & & & & ָั & $\simeq$ & & & $\wedge$ & 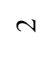 & \multirow{4}{*}{ 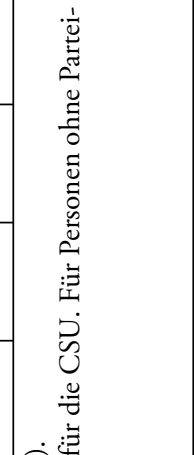 } \\
\hline & & & $m$ & $\simeq$ & & & & $\cong$ & $\stackrel{*}{n}$ & & & & & \\
\hline ปี & $\stackrel{2}{\omega}$ & 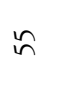 & i & สु & * & & & $\stackrel{\varpi}{\sim}$ & $\stackrel{n}{2}$ & & & त & $\stackrel{\infty}{-}$ & \\
\hline కี & ? & $a$ & $\stackrel{*}{\underset{\gamma}{\gamma}}$ & $\stackrel{\sim}{N}$ & & & & $n$ & ป & & & & $\stackrel{*}{\Lambda}$ & \\
\hline हू & 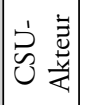 & 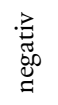 & 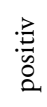 & 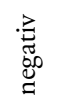 & 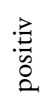 & & & 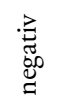 & $:$ & & & 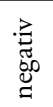 & $\begin{array}{l}: \\
0 \\
0 \\
0\end{array}$ & 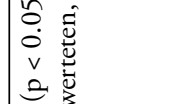 \\
\hline ह & 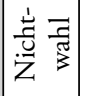 & $\stackrel{n}{n}$ & ㄱ. & ' & ' & $\stackrel{\sim}{\sim}$ & $\stackrel{*}{\infty}$ & $=$ & $\stackrel{\infty}{-}$ & 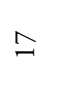 & & $\wedge$ & 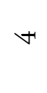 & 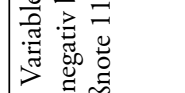 \\
\hline sँ & 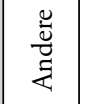 & $n$ & - & $\wedge$ & $\Xi$ & $\stackrel{\sim}{\simeq}$ & $n$ & ป & Fे & $\stackrel{\infty}{n}$ & $\stackrel{*}{\sim}$ & F & $\stackrel{\varpi}{\sim}$ & : \\
\hline 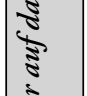 & $\hat{\hat{\omega}}$ & $\cong$ & $\underbrace{*}_{6}$ & $n$ & $\stackrel{*}{\stackrel{*}{F}}$ & $\infty$ & $\ddot{2}$ & $\sigma$ & กี & $\wedge$ & $\stackrel{*}{\infty}$ & $a$ & $\stackrel{*}{\stackrel{\sigma}{\sigma}}$ & 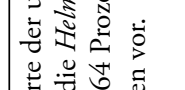 \\
\hline 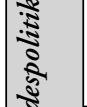 & Uु & $\underset{H}{*}$ & $\stackrel{*}{\beth}$ & ฉ & बे & 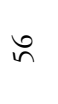 & $\stackrel{\sim}{\sim}$ & $\stackrel{\infty}{n}$ & $\stackrel{*}{\exists}$ & $\beth$ & ๙ & $\underset{F}{F}$ & $\tilde{\imath}$ & 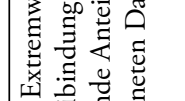 \\
\hline $\mid \begin{array}{l}0 \\
1 \\
5 \\
0\end{array}$ & 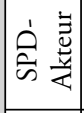 & 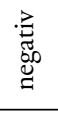 & $\begin{array}{l}: \\
: \\
0 \\
0 \\
0\end{array}$ & 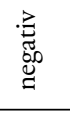 & 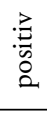 & 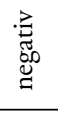 & $\begin{array}{l}: \geq \\
0 \\
0 \\
0 \\
2\end{array}$ & 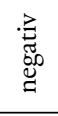 & $:$ & 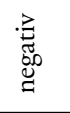 & 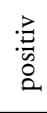 & 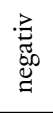 & 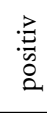 & 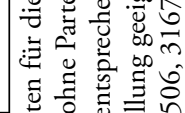 \\
\hline \begin{tabular}{|c}
5 \\
$\vdots$ \\
1 \\
1
\end{tabular} & 惫 & $\stackrel{0}{-}$ & $\stackrel{\sim}{\sim}$ & 1 & ' & $\underset{F}{\stackrel{H}{*}}$ & $\stackrel{\infty}{\sim}$ & $\Lambda$ & 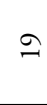 & $\vec{n}$ & $\wedge$ & $n$ & $n$ & 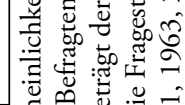 \\
\hline$\approx$ & $\frac{\tilde{u}}{\frac{u}{4}}$ & & $n$ & $\hat{\lambda}$ & $\checkmark$ & ปี & $N$ & $\hat{\wedge}$ & $\stackrel{*}{\sim}$ & $\stackrel{n}{~}$ & $\stackrel{\leftrightarrow}{+}$ & $\stackrel{n}{ }$ & ৯े & 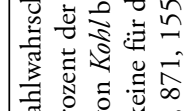 \\
\hline ปิ $จ$ & $\stackrel{\hat{\omega}}{\dot{\omega}}$ & $\infty$ & $\stackrel{*}{\beth}$ & $\stackrel{+r}{m}$ & 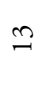 & $\vec{\sim}$ & $\sigma$ & 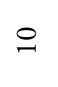 & 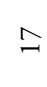 & $\vec{\sim}$ & ส & in & $\stackrel{*}{=}$ & 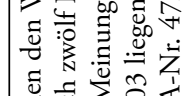 \\
\hline 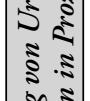 & $\stackrel{b}{0}$ & $\neg$ & $\stackrel{*}{\sim}$ & $\stackrel{\infty}{n}$ & $\stackrel{*}{*}$ & $\stackrel{\sim}{\simeq}$ & 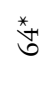 & $\wedge$ & $\stackrel{*}{\forall}$ & $\cong$ & $\curvearrowright$ & $\stackrel{\bullet}{-}$ & 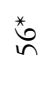 & 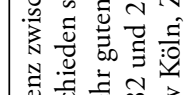 \\
\hline 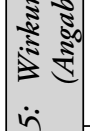 & 仓 & 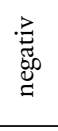 & 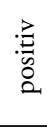 & 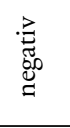 & 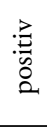 & 壹 & $\begin{array}{l}: \\
: \\
0 \\
0\end{array}$ & 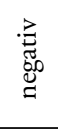 & $:$ & 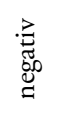 & 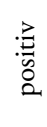 & 䔍 & : & 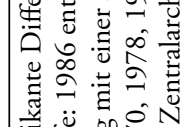 \\
\hline$\approx$ & $\frac{7}{\frac{\pi}{3}}$ & $\stackrel{\circ}{\precsim}$ & & $\stackrel{+}{\stackrel{2}{\Omega}}$ & & 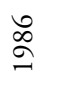 & & $\stackrel{\curvearrowright}{\cong}$ & & 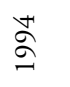 & & $\stackrel{\infty}{\check{\beth}}$ & & 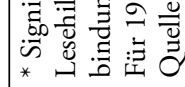 \\
\hline
\end{tabular}


Koalition bekämpfte, deutlich an Wirkung auf bayerisches Landtagswahlverhalten. Die prognostizierten Wahrscheinlichkeiten zeigen, dass Strauß' Wirkung sogar noch jene Brandts übertraf. Allerdings könnte beider Einfluss überschätzt sein, da die Befragung nicht unmittelbar vor der Landtagswahl 1974 stattfand, sondern im Frühsommer 1973. Theo Waigel war bei der Wahl 1990 vergleichsweise beliebt, doch ließen positive Urteile über den CSU-Vorsitzenden und Bundesfinanzminister die Wahrscheinlichkeit, in Bayern für die CSU zu stimmen, sinken, während sie die Wahlchancen der kleinen Parteien, und dabei speziell der FDP, steigerten. Diese erstaunlichen Befunde deuten darauf hin, dass der neue CSU-Vorsitzende knapp zwei Jahre nach der Amtsübernahme noch nicht vollkommen akzeptiert war, was mit seiner eher kooperativen denn konfrontativen Arbeit in der Bundesregierung zusammenhängen könnte. Bis zur Wahl 1998 änderte sich das. Nun steigerte Unterstützung für den CSU-Vorsitzenden die Chancen seiner Partei deutlich; allerdings war er mittlerweile ähnlich unbeliebt wie sein Vorgänger vor der Wahl 1974.

Wenn Bayerns Bürger über die Zusammensetzung des Landtags entscheiden, blicken sie also durchaus über die Grenzen ihres Freistaats hinaus. So wenig sie ein reines Landesvotum abgeben, so wenig ist ihre Wahlentscheidung jedoch vollständig von bundespolitischen Einstellungen determiniert. Im Gegenteil: Deren exklusiver Beitrag zur Erklärung von Landtagswahlverhalten lässt sich mit einstelligen Prozentsätzen angeben. Dies kontrastiert auffällig mit den Auskünften der Bürger über die Motive ihrer Wahlentscheidung: 1982 und 2003 gab rund die Hälfte der Befragten an, sie hätten bei der Wahlentscheidung eher die Bundes- als die Landespolitik im Sinn gehabt. Gefragt nach dem Stellenwert der Bundespolitik, billigten ihr 1994 und 1998 jeweils rund vier von fünf Antwortenden eine wichtige oder sehr wichtige Rolle zu. Eine solche Diskrepanz zwischen der Selbstwahrnehmung und der tatsächlichen Motivlage ist in der empirischen Sozialforschung nicht ungewöhnlich und daher an sich kein Anlass, die dargestellten Befunde in Zweifel zu ziehen.

Gleichwohl sprechen zwei Argumente dafür, dass die ausgewiesenen Bundeseffekte auf bayerisches Landtagswahlverhalten die untere Grenze des tatsächlichen Bundeseinflusses markieren: (1) Bundes- und landespolitische Einstellungen sind derartig eng verflochten, dass sich ihre Effekte auf das Wahlverhalten mit statistischen Mitteln nicht sauber trennen lassen. Wenn sich Bürger eine Meinung über Spitzenkandidaten zu einer Landtagswahl bilden, dürften sie kaum deren Äußerungen zu bundespolitischen Fragen ausblenden. Stellt sich nun heraus, dass diese Kandidatenorientierungen das Wahlverhalten beeinflussen, lässt sich nicht entscheiden, inwieweit es sich dabei um einen bundes- oder einen landespolitischen Effekt handelt. Der Erklärungsbeitrag nominell bundespolitischer Orientierungen unterschätzt daher in der Regel deren tatsächliche Wirkung. (2) In dieser Analyse konnten nur direkte Bundeseffekte, nicht aber indirekte untersucht werden. Damit blieben alle Reaktionen bayerischer Politiker auf bundespolitisches Geschehen, die in die Meinungsbildung der Bürger einfließen, ausgeblendet. Bedenkt man die Vielfalt potentieller indirekter Einflüsse - beispielsweise kann der bundespolitische Themenhaushalt die Streitfragen des Landtagswahlkampfes mitbestimmen, können Bundesparteien auf die Kandidatenauswahl im Land einwirken oder können landespolitische Koalitionsoptionen bundespolitisch vorgeformt sein -, ist diese Lücke nicht vernachlässigenswert klein. Daher ist erst recht davon auszugehen, dass die angegebenen Effekte die tatsächliche Bedeutung der Bundespolitik für bayerische Landtagswahlen unterschätzen. Wie groß der Bundeseinfluss unter Berücksichtigung dieser Argumente ist, lässt sich nicht exakt beziffern. Damit kann auch nicht entschieden werden, wie nahe die Selbsteinschätzung der Bürger den tatsächlichen Einflüssen kommt. 
Diese Argumente fallen in Bayern mindestens ebenso sehr ins Gewicht wie in anderen Bundesländern, und zwar hauptsächlich wegen der CSU. Sie ist eine bayerische Bundespartei, deren bundespolitische Bedeutung nicht zuletzt auf ihrem landespolitischen Nimbus beruht. Die Doppelrolle der CSU sorgt für eine institutionalisierte Vermischung von Bundesund Landespolitik, wofür bayerische Ministerpräsidenten auf der Position des CSU-Vorsitzenden das eindrücklichste Beispiel liefern. ${ }^{14}$ Wenn die CSU-Führung bundespolitisch Stellung bezieht, handelt die bayerische Regierungspartei. Die bayerische Regierung kann bundespolitische Ziele der CSU verfolgen - man denke an Klagen vor dem Bundesverfassungsgericht oder den Bundesrat, wie umgekehrt die CSU im Bund bayerische Interessen fördern kann. Dieses komplizierte Wechselspiel zwischen den politischen Arenen wird zudem vom Imperativ, die Mehrheit in Bayern zu halten, geprägt. Er legt es der CSU nahe, sich landespolitisch von bundespolitischen Akteuren zu distanzieren, mögen diese auch einem Koalitionspartner (oder der eigenen Partei) angehören. Diese Strategie begünstigt nicht nur ungewöhnliche Effekte bundespolitischer Orientierungen auf Landtagswahlverhalten, sondern auch die Vermischung von Bundes- und Landespolitik. Insgesamt dürften in Bayern daher besonders starke indirekte bundespolitische Effekte auf Landtagswahlverhalten auftreten, wie es auch noch häufiger als in anderen Ländern sehr schwierig sein dürfte, bundes- und landespolitische Einflüsse zu entwirren. Paradoxerweise scheinen die genuin bayerische CSU und die von ihr betonte Sonderstellung Bayerns bundespolitische Einflüsse auf bayerische Landtagswahlen nicht zu schwächen, sondern eher zu stärken.

\section{Zusammenfassung}

(1) Parteibindungen und landespolitische Orientierungen waren zwischen 1966 und 2003 in Bayern in der Regel deutlich zugunsten der CSU verteilt. Ähnliches gilt in vielen Fällen für bundespolitische Einstellungen; vor den Wahlen 1974 und 1998 war dies jedoch nicht der Fall.

(2) Bundespolitische Orientierungen haben das individuelle Verhalten bei bayerischen Landtagswahlen beeinflusst. Einstellungen zur Arbeit der Bundesregierung fallen dabei kaum ins Gewicht. Wichtiger sind Urteile über Bundespolitiker.

(3) Gemessen am statistischen Erklärungsbeitrag bundespolitischer Orientierungen ist der Bundeseinfluss auf bayerisches Landtagswahlverhalten sehr klein. Damit wird die tatsächliche Bedeutung der Bundespolitik jedoch unterschätzt, da die Vermischung von Landes- und Bundespolitik sowie indirekte Bundeseffekte unberücksichtigt bleiben.

(4) Die Doppelrolle der CSU als bayerische Bundespartei begünstigt die Vermischung von Bundes- und Landespolitik sowie bundespolitische Einflüsse auf bayerisches Landtagswahlverhalten. Überspitzt formuliert, könnte eine Besonderheit bayerischer Landtagswahlen somit in ihrer vergleichsweise starken bundespolitischen Durchdringung liegen.

14 Siehe etwa Rainer-Olaf Schultze, Die bayerische Landtagswahl vom 12. Oktober 1986: Stabile Verhältnisse wie nachhaltige Veränderungen, in: ZParl, 18. Jg. (1987), H. 1, S. 38 - 56; ders., Die bayerische Landtagswahl vom 14. Oktober 1990: Bayerische Besonderheiten und bundesrepublikanische Normalität, in: ZParl, 22. Jg. (1991), H. 1, S. 40 - 58. 